\title{
Réalisation de plaques tournantes Plaque de Grenoble
}

\author{
Construction of rotating slabs \\ Grenoble model
}

\author{
PAR J. DALVERNY ET F. FONTANET
}

INGÉNIEURS A ra SOGREAH, GRENOBLE

\begin{abstract}
La première partie de la communication est consacrèe à l'examen des phénomènes parasites qui peavent être induits sur le modèle par l'irrégularité du mouvement de rotation de la plaque. On en déduit l'ordre de grandeur de la précision exigée pour l'usinage, la pose, et la conservation du chemin de ronlement et pour le controle de la vitesse de rotation.

La deaxième partie est consacrée à la description de deux solutions originales étudiées par le Laboratoire Dauphinois d'Hydraulique pour le compte de l'Université de Grenoble.

- Modele flottant, guidé par un chemin de roulement très pen chargé (solution abandonnée);

- Plaque rigide, guidé par un chemin de ronlement et portee par un pioot central, monte sur crapaudine iprojet en cours de réalisation).
\end{abstract}

La rentabilité des usines marémotrices dipendant essentiellement de l'importance des courants et des marnages au droit de l'usine, il est indispensable, lorsqu'on établit l'avant-projet d'une telle usine, de connaittre sa réaction sur la marée, pour diverses conditions d'exploitation.

On conçoit donc l'intérêt que présente la construction d'un modèle réduit permettant la reproduction fidèle du mouvement de la mer sur des superficies de quelque importance.

On sait, d'autre part, que la rotation de la terre joue un rôle de premier plan dans l'explication des marées, notamment dans la Manche occidentale. L'étude de l'aménagement des îles Chausey dans le fond du golfe de SaintMalo nécessitait donc la construction d'un grand modèle tournant, permettant de reproduire la rotation de la terre, à l'échelle du modèle.

La réalisation de ce modèle - le plus grand du monde - a été décidée par l'Université de Grenoble, qui dispose par ailleurs du concours du Laboratoire Dauphinois d'Hydraulique et du Laboratoire National de Chatou. L'étude du pro-

\begin{abstract}
The first part of the paper examines stray effects which uneven rotation of the lable might set up in the model. Deductions are made from this concerning the amount of precision required during machining, erection and maintenance of the roller track and concerning the accuracy with which the rotational speed has to be maintained.

The second part consists of a description of the following two designs prepared by the Laboratoire Dauphinois d'Hydraulique on behalf of the lniversity of Grenoble:

a) a floating model guided by a roller tracl: carrying a bery small load (discarded design),

b) a rigid table guided by a roller track and borne by a centrally located step-bearing (model now being built).
\end{abstract}

jet et son exécution ont été confiées au Laboratoire Dauphinois d'Hydraulique.

Pour donner une idée de ses dimensions (fig. 1),

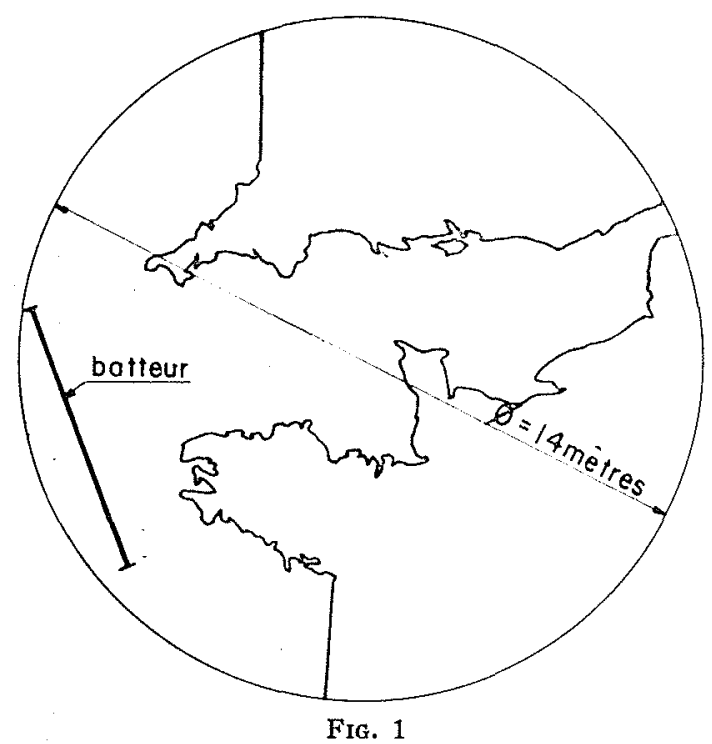

Plan du modèle de la Manche au 1/50000\% 
disons simplement que la Manche entière, reproduite à l'échelle du $1 / 50000^{\circ}$ en plan, occupera une plateforme de $14 \mathrm{~m}$ de diamètre. Cette plateforme effectuera un tour complet en 50 secondes. Elle pèsera 250 tonnes environ.

Les projets de construction d'une plaque tournante de cette dimension peuvent présenter de nombreuses variantes dans les détails d'exécution, mais la conception d'ensemble reste la même. On réalisera une plateforme en béton armé, tournant autour d'un pivot central et s'appuyant sur un chemin de roulement de grand diamètre.

Avant d'aborder l'étude du projet proprement dit, il importait d'examiner les phénomènes parasites qui pourraient être induits sur le modèle par les inévitables irrégularités du mouvement. Il importait aussi d'évaluer l'ordre de grandeur de ces phénomènes, afin de fixer les marges de lolérance qui pourraient être admises dans la réalisation du chemin de roulement ei du système d'entraînement.

Les perturbations qui nous ont paru les plus redoutables peuvent provenir des défauts suivants :

1. Irrégularité de la vitesse de rotation;

2" Défaut d'horizontalité ou de planéité du chemin de roulement;

$3^{\circ}$ Défaut d'équilibrage statique et dynamique de la plaque.

Examinons-les successivement :

\section{Irrégularité de la vitesse de rotation.}

Lorsque la masse d'eau est en repos relatif, la surface libre prend la forme d'un paraboloïde d'équation :

$$
z=-\frac{\omega_{0}^{2} l^{2}}{2 g}
$$

(fig. 2).

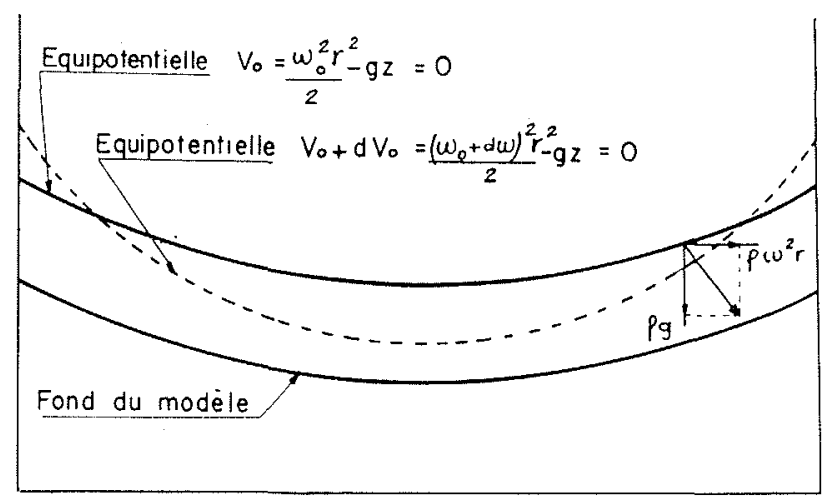

FIG. 2
Ce paraboioïde représente le niveau zéro des mers et c'est à ce niveau de référence que seront rapportées non seulement les mesures de marnages, mais aussi les courbes de niveau du fond. $\mathrm{Si}$, en cours d'exploitation, la vitesse de rotation. est différente de la valeur $\omega_{0}$ pour laquelle le modèle a été construit, la parabolö̈de change de forme et les profondeurs d'eau au centre ou sur les bords ne correspondent plus aux profondeurs réelles.

Si la masse d'eau recouvre toute la plaque, on trouve qu'un écart de $1 \%$ sur la vitesse $\omega_{0}$ entraîne un écart de $4 / 10^{\circ}$ de millimètre sur la cote du plan d'ean au centre et sur les bords.

Dans le cas de variations lentes de la vitesse de rotation, le niveau varie lentement et d'une quantité faible qui n'est pas gênante. Mais dans le cas de variations périodiques, il peut se créer dans le modèle une oscillation forcée, qui se superpose à la marée, et qui fausse notablement les mesures. En effet, avec une échelle rerticale de $1 / 500^{\circ}$, ce qui représente déjà une distorsion importante de l'échelle des hauteurs (distorsion $=(1 / 500) /(1 / 50000)=100)$, les marnages seront de l'ordre de 1 à $2 \mathrm{~cm}$ seulement et une oscillation parasite de $2 \mathrm{~mm}$ peut représenter une srreur de 10 à $20 \%$.

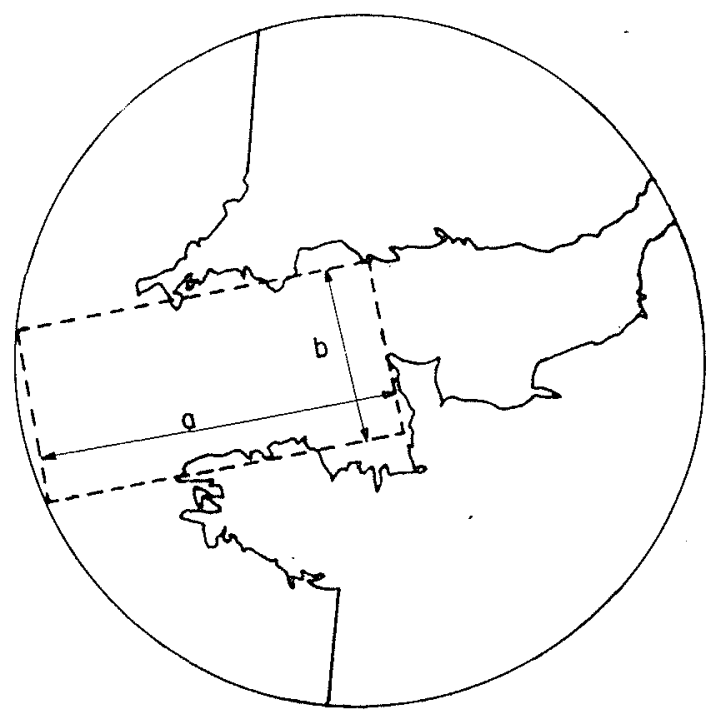

FIG. 3

Pour évaluer l'ordre de grandeur de ces oscillations, on doit se contenter de calculs simplifiés, faute de savoir intégrer exactement les équations du mouvement.

Nous avons schématisé la Manche par un bassin rectangulaire de profondeur $h$, de longueur $a$ et de largeur $b$, disposé selon un rayon de la plaque (fig. 3). Nous avons admis que le nouve- 
ment de l'eau pourrait ètre représenté par une seiche d'équation :

$$
\mathrm{U}=\mathrm{A} \sqrt{\frac{g}{a}} \sin \frac{\pi x}{a} \cos \frac{\pi \sqrt{g h}}{a} t
$$

U étant le courant et A la demi-amplitude de la seiche.

En fait, lorsque le canal tourne, les ondes à crète horizontale sont remplacées par des ondes de Kelvin, pour lesquelles la distribution des courants selon la largeur est exponentielle; ceci modifie notablement l'évaluation des frottements, tout au moins lorsqu'ils sont quadratiques. Puisque nous ne recherchons qu'un ordre de grandeur, nous nous contenterons cependant de cette approximation.

On peut exprimer les condilions d'entretien du mouvement en écrivant que l'énergie dissipée par le frottement est fournie par le travail des forces appliquées. Ce dernier peut s'évaluer simplement de la manière suivante :

Lorsque la masse d'eau est en repos relatif, la force centrifuge par unité de volume $\left(\rho(\omega)^{2} r\right)$ est équilibrée par le gradient de pression (fig. 4).

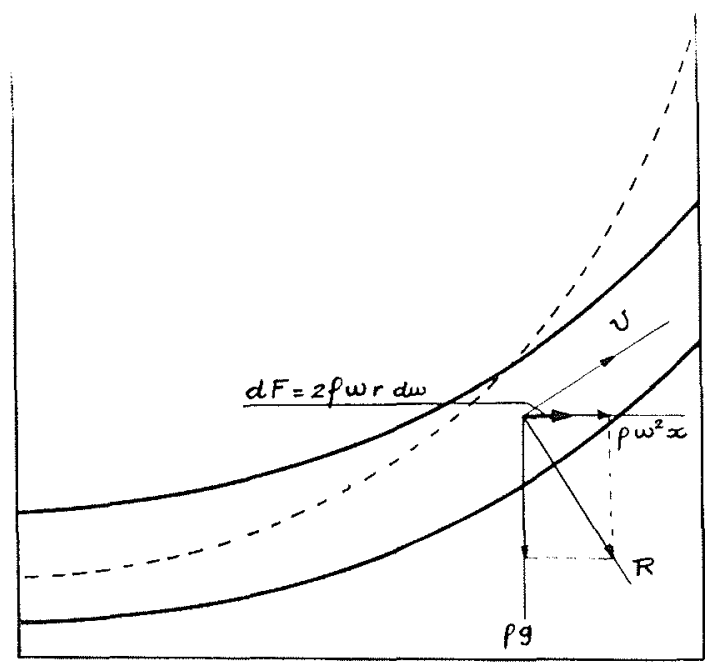

Fig. 4

Si $\omega$ varie de $d \omega=\Delta \omega \cos (\pi \sqrt{g h} / a) t$, la force centrifuge varie d'une quantité :

$$
d \mathrm{~F}=2 \rho_{(1)} r d(\omega)=2 \rho(1) r \Delta(1) \cos (\pi \sqrt{g h} / a) l,
$$

qui constitue la force excitatrice. A la résonance, cette force est constamment en phase avec le courant, et l'énergie fournie au fluide pendant une période est donnée par l'intégrale $\left(^{*}\right)$ :

$\left(^{*}\right)$ On confond $d F$ avec sa projection sur $U$, et $r$ avec labscisse curviligne du point.

$$
\begin{array}{r}
E_{1}=\int_{0}^{a} \int_{0}^{\prime \prime}\left(2 \rho \omega r \Delta \omega \cos \frac{\pi \sqrt{g h}}{a} l\right) b h d r \mathrm{U} d t \\
=\frac{(\omega) \Delta \omega \mathrm{A} a^{*} b}{2 \pi}
\end{array}
$$

La force de Coriolis ne travaille pas. Il en est de mềme de la pesanteur et de la force centrifuge moyenne $\omega^{2} r$, dont la résultante $R$ est perpendiculaire au courant.

L'énergie dissipée par le frottement est facile à calculer si on connait la loi de frottement.

En admetant un frottement quadratique, la force de frottement par unité de surface es! donnée par $\tau=-f|u| u$ et l'on at:

$$
E_{2}=\int_{0}^{* \prime} \int_{0}^{\top T}(f|u| u b d r) u d t=\frac{32}{9} \frac{f a^{2} g A^{\prime \prime} \mid}{h^{2} a^{2}}
$$

Si l'on admet, par contre, un frottement linéaire $\tau=-\mathrm{F} u$, on a :

$$
\mathrm{E}_{3}=\int_{0}^{a} \int_{0}^{\mathrm{T}}(\mathrm{F} u b d r) u d t=\frac{\mathrm{F} a^{2} \mathrm{~A}^{2} b}{2 h} \sqrt{\frac{g}{h}}
$$

En égalant $E_{1}$ et $E_{2}$ ou $E_{1}$ et $E_{3}$, on trouve les formules :

1. Frottement Quadratique:

$$
\frac{\Delta \omega}{\omega}=\frac{16}{9}-\frac{f g}{a \pi \rho} \frac{(2 \mathrm{~A})^{2}}{h^{2} \omega^{2}}
$$

\section{Frottement linéaire :}

$$
\frac{\Delta \omega}{\omega}=\frac{\pi \mathrm{F}}{2 a h \omega^{2} p} \sqrt{\frac{g}{h}}(2 \mathrm{~A})
$$

Pour faire l'application numérique, on remarque que l'oscillation qui s'installe entre le bord du modèle où sera disposé le batteur et le centre où se trouvera la presqu'île du Cotentin, est seule partout en phase avec la force excitatrice. C'est done celle-ci qui se produira normalement. On prendra par suite $a=7 \mathrm{~m}, h=0,10 \mathrm{~m}$. Quant au coefficient de frottement, il est très incertain.

La valeur donnée par Hansen pour la Manche correspond dans la nature à $f=2,3 \times 10^{-3}$ tonnes par metre cube (frottement quadratique). Sur le modele, le coefficient de frottement sera cent fois plus fort si la distorsion est de 100. On devra prendre $f=0,23$. Si l'on veut limiter l'amplitude de la seiche à $2 \mathrm{~mm}(2 \mathrm{~A}=2 \mathrm{~mm})$, il ne faut pas tolérer d'écarts de vitesse supérieurs à :

$$
\frac{\Delta \omega}{\omega}=0,5 \%
$$

En fait, poux un mouvement d'aussi faible amplitude, le nombre de Reynolds calculé en pre- 
nant la profondeur comme dimension caractéristique, est de l'ordre de 1000 . Le mouvement sera probablement laminaire. En admettant que la loi linéaire se raccorde avec la loi quadratique pour un nombre de Reynolds de 3000 , on obtient pour le coefficient $F$ une valeur égale à $7 \times 10^{-3}$ (tonnes par mètre carré et par seconde).

On a dans ces conditions, pour $2 \mathrm{~A}=2 \mathrm{~mm}$ :

$$
\frac{\Delta(1)}{\omega}=2 \%
$$

Ces ordres de grandeur correspondent à l'oscillation qui se produit lorsque le modèle tourne, le batteur étant lui-même arrêté. Lorsque le batteur fonctionne, le courant a la forme $\mathrm{U}+u$, $\mathrm{U}$ représentant la vitesse qui serait observée si la rotation était parfaitement régulière et $u$ représentant l'oscillation parasite. On peut étudier cette dernière séparément à la condition de prendre pour expression du frottement $\tau=-2 f|\mathrm{U}| u$ (coefficient de frottement de Boussinesq, égal à la dérivée de la force de frottement du mouvement général).

En prenant pour U la vitesse moyenne $0,05 \mathrm{~m}$ par seconde, on a un coefficient de frottement linéaire de 0,023 , trois fois plus fort que le coefficient précédent. Pour créer une seiche de $2 \mathrm{~mm}$, il faudrait donc des écarts de $6 \%$ sur la vitesse de rotation, et cela dans le cas de résonance.

On étudierait de façon analogue l'influence des accélérations tangentielles.

On pourrait penser d'autre part que la seule inertie du modèle suffirait à amortir les varia-

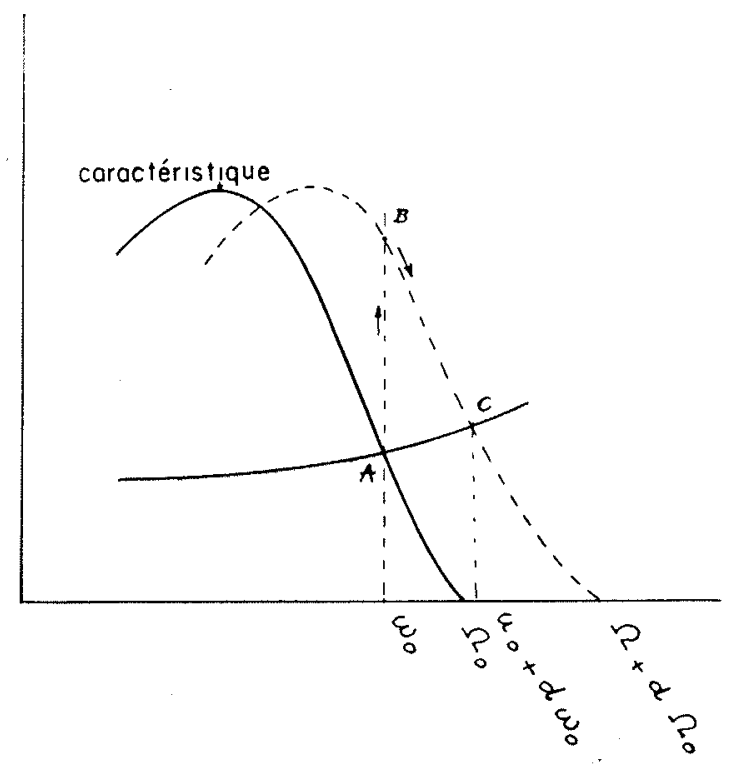

Fici. 5 tions brusques de la vitesse de rotation. Nous avons donc cherché à évaluer le temps de réponse du système d'entrainement lorsque la fréquence du secteur varie brusquement (fig. 5).

La vitesse de rotation $\omega_{0}$ du moteur est définie par l'intersection $A$ de la courbe caractérisque donnant le couple en fonction du glissement, et de la courbe donnant le couple résistant en fonction de la vitesse de rotation.

Si la fréquence du secteur varie de $+\Delta \Omega_{0}$, la caractéristique se déplace vers la droite, le couple moteur devient supérieur au couple résistant (point B) et le mouvement s'accélère. Ceci a pour efret de diminuer le glissement et par conséqueni le couple moteur, alors que le couple résistant augmente légèrement. La nouvelle vitesse de régime sera atteinte en C. En intégrant l'ćquation :

$$
I \frac{d \omega}{d t}=\mathrm{C}_{m}(\omega)-\mathrm{C}_{r}(\omega)
$$

nous avons déterminé le temps nécessaire pour passer du point A au point C. Il est de l'ordre de la seconde, pour les valeurs du couple moteur et du couple résistant que nous avons calculées. Ainsi le modèle suit pratiquement la fréquence du secteur, l'inertie de la plaque n'intervenant pas.

On peut faire un calcul analogue pour apprécier l'influence des variations de tension sur le glissement du moteur.

En résumé, la fréquence et la tension du secteur étant relativement bien définies, les irrég!1larités de la vitesse de rotation ne seront probablement gênantes que pour les réglages de niveau exécutés batteur arrêté. Cependant, afin d'éliminer toute difficulté et aussi afin de faciliter le lancement du modèle et son entrainement par plusieurs moteurs, nous avons prévu une alimentation en courant continu avec un contrôle tachymétrique assurant une grande régularité de la vitesse de rotation.

\section{Défaut d'horizontalité et de planéité du chemin de roulement.}

Un système d'axes liés à la plaque XOY peut ctre défini par rapport aux axes fixes $x$ Oy par les angles d'Fuler 0, w $t, i$ (fig. 6 ).

Les composantes de la pesanteur dans le système d'axes liés sont:

$$
-g g \sin i \sin \omega t \quad g g \sin i \cos \omega t \quad-g g \cos i
$$

Si, par suite d'un défaut de construction ou d'un léger affaissement des fondations, le chemin de roulement présente une inclinaison $i$ par rapport au plan horizontal (ce qui correspond au cas où 0 et $i$ sont constants), la masse d'eau sera sollicitéc dans le mouvement relatif par un champ 


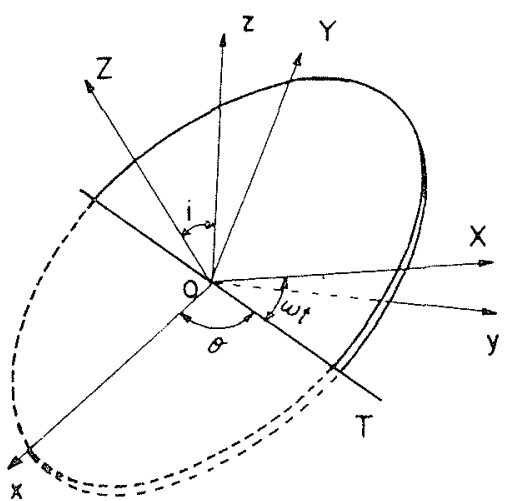

Fig. 6

de forces tournant, parallèle au fond. Il en résultera des oscillations longitudinales et tranversales de périodes :

$$
\frac{2 \pi}{\omega}
$$

De même, si le chemin de roulement n'est pas plan, les angles 0 et $i$ varieront au cours de la rotation et seront fonction périodique de

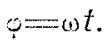

On montre ainsi que les forces excitatrices se composent d'un certain nombre d'harmoniques de la fréquence fondamentale $\omega$.

On peut évaluer l'importance des seiches créées par ces champs de forces parasites en établissant comme ci-dessus le bilan de l'énersie apportée au fluide et de l'énergie consommée par frottement.

Dans le cas de résonance, où la force est en phase avec le courant, on calcule sans difficulté l’énersie apportée pendant une période:

$$
\begin{array}{r}
\mathrm{E}_{1}=\int_{n}^{\mathrm{T}} \int_{a}^{a}\left(g i \cos \frac{\pi \sqrt{g h}}{a} t h b d x\right) u d t \\
=\frac{2 \rho g i \mathrm{~A}\left(i^{2} b\right.}{\pi}
\end{array}
$$

d'oì les équations définissant $i$ :

1" Frotoment guadratigue:

$$
i_{1}=\frac{4 f(2 \mathrm{~A})^{2}}{9 h^{2} \pi ?}
$$

2" Frotthement linéaire:

$$
i_{2}=\frac{F(2 A)}{8 g h \sqrt{g h}}
$$

On aura donc, pour $2 A=2 \mathrm{~mm}$ :

$$
i_{1}=1,3 \times 10^{-5} \text { et } i_{2}=5,5 \times 10^{-5}
$$

Cette inclinaison correspond à un défaut de nivellement du chemin de roulement égal à $\varepsilon=i \mathrm{R}$, scit respectivement $1 / 10^{\circ}$ et $3 / 10^{\circ}$ de $\mathrm{mm}$.

Ces conditions sont extrêmement sévères et paraissent à première vue devoir condamner la réalisation d'un tel projet. En réalité, il convient de remarquer que la résonance ne sera vraisemblablement pas réalisce, et que pendant T'exploitation du modele, Ie coefficient de frottement lineaire apparent: $2 f|\mathrm{U}|$ sera en réalité trois fois plus fort que le coefficient $F$. Une seiche de $2 \mathrm{~mm}$ correspondra en cas de résonance à un défaut de nivellement de $1 \mathrm{~mm}$ sur le chemin de roulement.

Enfin, l'amplitude de la perturbation ne sera égale à 2 mm qu'au ventre de la seiche.

On pourrait envisager d'enregistrer les seiches à part afin de les extraire de la courbe d'enregistrement totale. Mais les phénomènes n'étant pas linéaires, la correction serait inceriaine.

\section{Défaut d'équilibrage statique et dynamique.}

INFLUTNCE DU COUPLE GYROSCOPIQUE:

Le modèle constitue une masse importante inégalement répartie. Il paraît difficile de l'équilibrer parfaitement, même si l'on dispose judicieusement le générateur de marée et les diver's appareils tournant avec le modèle. On ne pourrait d'ailleurs réaliser approximativement l'équilibrage que par le calcul.

Nous avons done évalué les efforts anormaux qui seront supportés par le pivot et le chemin de roulement et qui seront dus aux défauts d'équilibrage statique et dynamique, ainsi qu'au couple syroscopique. Nous avons trouvé des efforts inférieurs à la centaine de kilos, ce qui ne paraît donc pas devoir être gênant.

\section{ETUDE DU PROJET :}

Les marges de tolérance étant ainsi fixees, nous pouvions passer à l'étude du projet.

Le probleme consistait à faire tourner une masse de 200 à 300 tonnes avec une précision d'horlogerie.

Le chemin de roulement devait être construit avec le plus grand soin et ne pas présenter de flèche appréciable au passage d'un galet. Mais, de plus, il devait conserver sa cote d'origine, en dépit des inévitables tassements des fondations. Il fallait donc non seulement s'assurer, par des sondages, de la stabilité des terrains et réaliser d'importantes fondations, mais il fallait aussi chercher une solution originale, dans laquelle le chemin de rovlement serait utilisé pour gui- 
der le modèle plutôt que pour le porter. Enfin il fallait prévoir des systèmes de réglage, qui permettraient de corriger les tassements du terrain ou le fluage des bétons.

La première solution, suggérée par M. Danel, consistait à faire flotter le modèe soit comme un bateau, soit comme la cloche d'un gazometre (fig. 7). Cette deuxieme formule présentait l'avantage, par rapport à la première, de mettre plus facilement la pivoterie à l'abri de l'eau et des sables. Elle permettait également, le cas
Nous avons finalement abandonné cette solulion, car nous n'avons pas su résoudre convenablement le problème du contrôle de la température sur les deux faces de la dalle. En effet, un calcul simple nous a montré qu'une dalle mince supportée sur toute sa surface subissait une importante déformation sous l'influence d'un gradient de température (un quart de centimètre de fleche par degré pour une dalle de $10 \mathrm{~cm}$ d'épaisseur). Ces déformations auraient amené d'importantes variations de profondeur

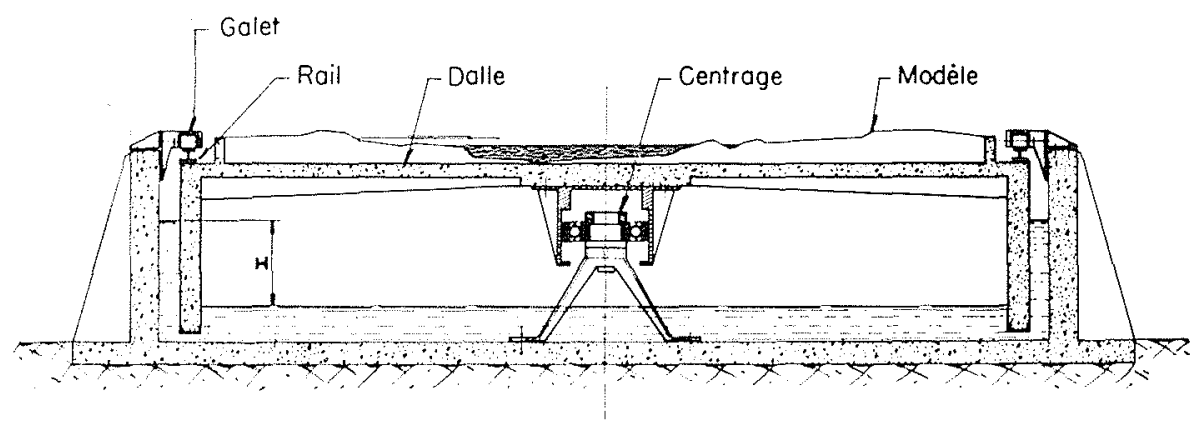

Fici. 7

Schema d'une dalle flottante

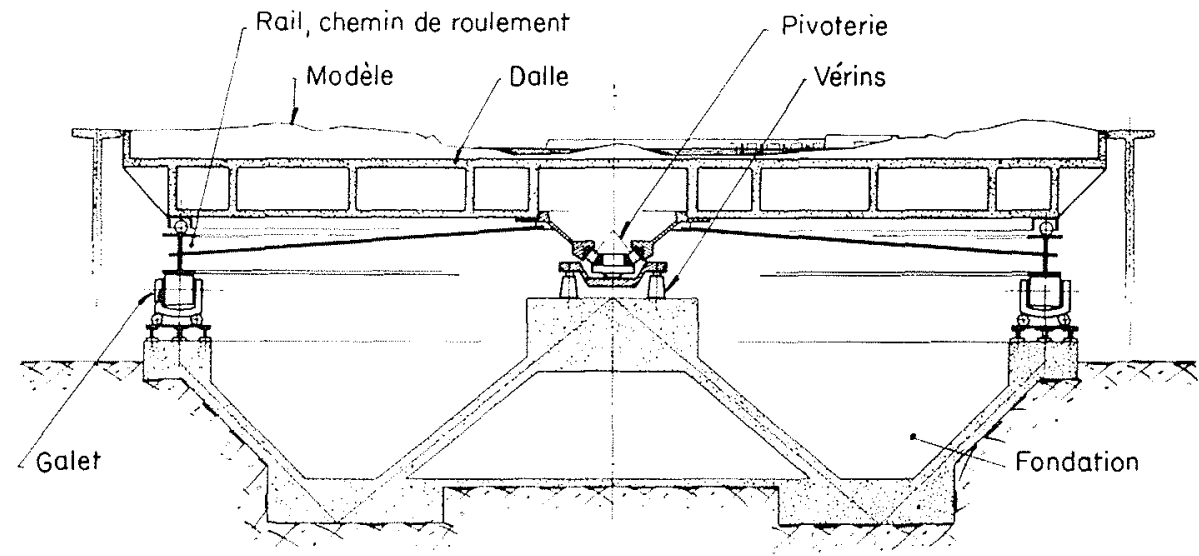

Fic, 8

Schéma d'une dalle supportée par un pivot

échéant, d'effectuer des réparations sous la dalle, ce qui était exclu dans le premier cas. On pensail d'ailleur's communiquer un excès de poussée au modèle, celui-ci élant maintenu partiellement enfoncé par le chemin de roulement. Cette disposition permettait de laisser reposer la plaque sur le fond, pendant l'arrêt du travail. On avait prévu un contrôle de la pression d'air pour se prémunir contre les fuites à travers les fentes de la dalle, et egalement contre les variations de poussées dues aux changements de température de la masse d'air. sur le modèle, et par suite des écarts inacceplables sur la célérité des ondes. On aurait pu, il est vrai, réduire les déformations en nervurant la plaque mais alors son poids aurait été trop grand pour qu'elle puisse flotter.

Nous sommes donc revenus à une conception plus classique. Ce deuxième projet, dont la réalisation est commencée, a été étudié également par MM. Angles d'Auriac, Desplats et Maneglia (fig. 8).

La plaque est supportée cette fois-ci par un pirot monté sur rotule. La rotule repose elle- 
mème sur des vérins qui permeltront de rattraper les tassements du terrain et le fluage du béton, mais qui permettront aussi de régler le partage du poids de la dalle entre le pivot et 12 galets disposés à la périphérie. La dalle s'appuiera sur ces galets par l'intermédiaire d'un chemin de roulement de grande dimension. Si l'on soulevait suffisamment la pivoterie, la dalle serait portée par le pivot seul et prendrait sous son propre poids (130 tonnes) et sous une charge de 170 tonnes, une lleche de l'ordre de $2 \mathrm{~cm}$. On réglera la fleche de maniere a laisser aux galets une charge totale de 60 à 100 tonnes.

Nous allons maintenant passer rapidement en revue les différents constituants de l'ensemble:

\section{Fondations :}

On s'est attaché à réaliser des fondations rigides et monobloc. On évite ainsi les mouvements relatifs du pivot et de la couronne poite-galets. La construction en double còne donne une rigidité remarquable eu égard au poids du béton. Celui-ci a été calculé de manière à faire supporter au sol un poids égal à celui de la terre enlevée, afin de conserver autant que possible l'équilibre naturel des terrains.

\section{Chemin de Roulement :}

Le chemin de roulement est constitué par 20 éléments de fer en I de 600 , formant un polygone de 6 m d'apothème. Les dimensions des fers ont été déterminées de facon à limiter à quelques centièmes de $\mathrm{mm}$ la flèche du rail, entre deux galets. Soigneusement usinés, ces rails ont été assemblés deux par deux, sur un marbre de précision. Le défaut de planéité obtenu sur deux éléments contigus est inférieur à $5 / 10^{\text {èmes }}$ de $\mathrm{mm}$. Des clavettes de positionnement légèrement forcées permettront d'assurer sur place un montage aussi parfait que possible. Le chemin de roulement tout entier présentera cependant des défauts plus importants, mais son poids et sa charge le maintiendront en contact avec les galets. Le défaut de planéité du chemin de roulement tout entier se traduira donc essentiellement par des variations de la charge supporlće par les galcts.

\section{GALETS:}

Ceux-ci pourront être réglés en hauteur avec une grande précision de façon à définir le plan horizontal avec le minimum d'erreurs.

Afin de répartir au mieux les efforts sur les deux paliers dans le cas où la surface du rail serait légèrement gauche, on a placé les galets sur un berceau qui peut rouler sur quatre roulements montés sur deux axes. Le centre de rota-

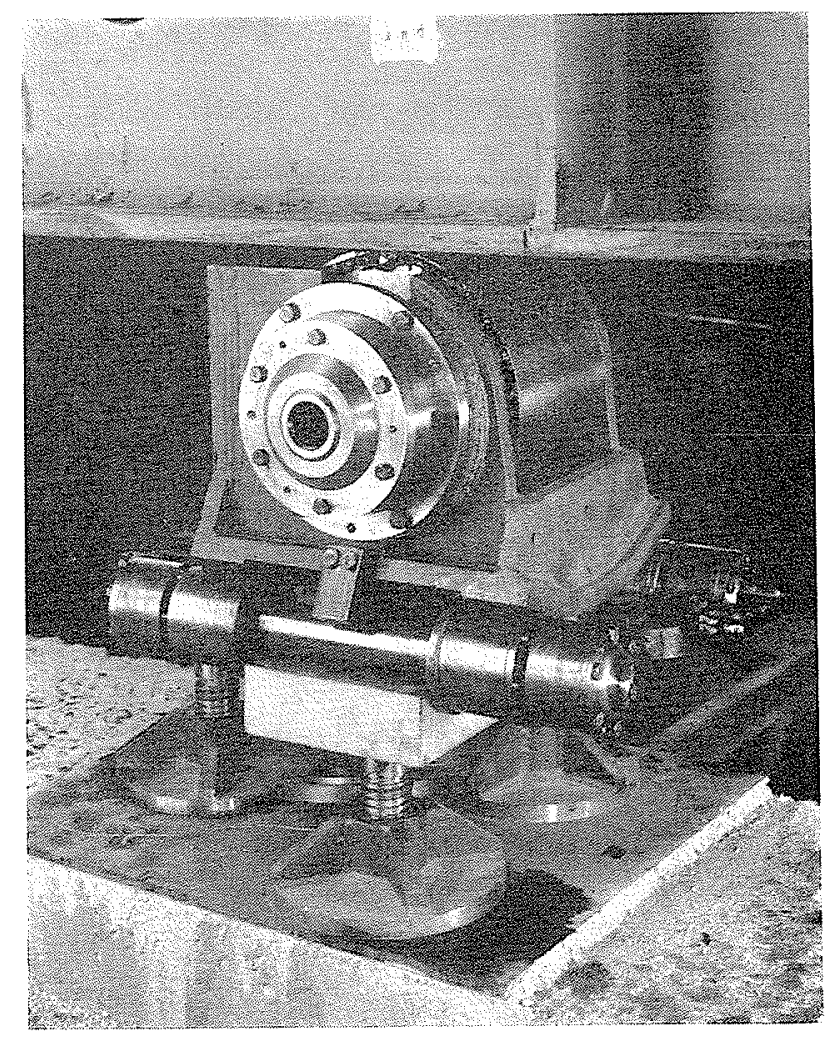

Photo 3

Vue d'un galet monté.

Lion du berceau est situé au milieu de la ligne de contact rail-galet, de sorte que son mouvement n'entraine pas de déplacement vertical.

Le support du berceau est monté sur trois vérins assurant le réglage en hauteur. Ceux-ci sont reliés par deux anneaux dynamométriques actionnant un circuit en cas de variation excessive de la charge.

Enfin, les galets comportent un réglage radial. Six d'entre eux sont moteurs et assurent l'entrainement de la plaque.

\section{Pivoterite:}

Elle est calculée pour supporter 300 tonnes à la vilesse de 4 tours à la minule. Elle est liee au rail par des rayons.

Un collecteur électrique assure la distribution du courant aux appareils situés sur la plateforme.

\section{DALLL:}

Elle est formée par deux voiles de béton armé réunis par des poutres en quadrillage. La conception béton armé nous a semblé préférable, pour plusieurs raisons, dont les principales sont une meilleure inertie thermique et un prix avanlageux. 
La dalle repose sur le rail par l'intermédiaire de rouleaux laissant du jeu pour la dilatation. Enfin, sa face inférieure sera ventilée avec de l'air à la température ambiante, de facon à éviter les déformations qui résultent des gradients de température.

\section{Mécanisme d'entraînement :}

Six galets seront entraînés par des moteur's à courant continu, à excitation séparée. Ils sont commandés par groupe de trois par deux amplidynes. I_e système d'entrainement est asservi. Un discriminateur de phase compare l'écart existant entre un générateur pilote à basse fréquence et un alternateur entraîné par la plaque. Il fournit un signal d'erreur sous la forme d'une tension proportionnelle à l'écart de phase des deux tensions alternatives.

Les premiers essais ont donné une précision d'affichage de $1 \%$ et semblent devoir assurer une régulation de vitesse à $0,2 \%$.

$$
\text { 象 }
$$

Nous espérons ainsi que les précautions prises, tant dans la conception que dans l'exécution de ce projet, nous permettront de surmonter les difficultés mises en évidence au cours de la première partie de cet exposé.

Cette belle réalisation permettra ainsi de traiter avec une bonne précision les nombreux problèmes qui vont se poser dans l'étude de l'aménagement des îles Chausey et même peut être de quelques autres usines marémotrices projetées à l'étranger. Elle sera de plus un magnifique instrument de recherche qui sera utilisé par les élèves de la Faculté des Sciences de Grenoble Iravaillant sous la direction de MM. les Professeurs Kravtchenko et Santon, et de M. Chabert d'Hières. Elle contribuera donc également au progrès de la Science en permettant d'approfondir notre connaissance de l'hydrodynamique des systèmes tournants.

\section{COMMENTAIRE}

de M. Ie Professeur Lacombe,

Président de séance.

M. le Président remercie M. Fon'raner d'avoir présenté cette communication.

If félicite MM. Darvenxy et Fontanet, ainsi que toute l'équipe du Laboratoire Dauphinois d'Hydraulique, d'avoir conçu et examiné ce système mécanique avec les infinies précautions qu'il exigeait.

Il souhaite bonne chance a cette plaque, pour sa vitesse de rotation et ses caractéristiques dynamiques et statiques.

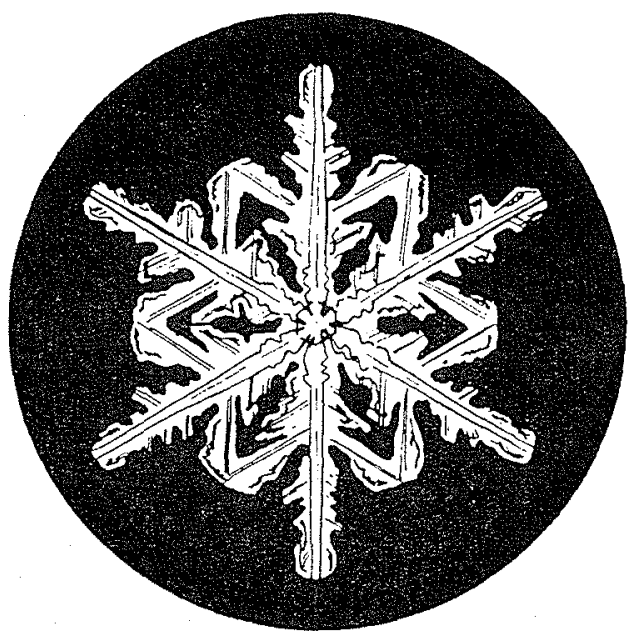

